\title{
Affective Guide with Attitude
}

\author{
Mei Yii Lim, Ruth Aylett, and Christian Martyn Jones \\ School of Mathematical and Computer Sciences, \\ Heriot Watt University, \\ Edinburgh, EH14 4AS, Scotland \\ $\{$ myl, ruth, cmj\}@macs.hw.ac.uk
}

\begin{abstract}
The Affective Guide System is a mobile context-aware and spatial-aware system, offering the user with an affective multimodal interaction interface. The system takes advantage of the current mobile and wireless technologies. It includes an 'affective guide with attitude' that links its memories and visitor's interest to the spatial location so that stories are relevant to what can be immediately seen. This paper presents a review of related work, the system in detail, challenges and the future work to be carried out.
\end{abstract}

\section{Introduction}

The main aim of this research is the creation of an 'affective guide with attitude' to provide adaptive guidance and engaging interaction. Here, the word 'attitude' refers to the guide's perspective and personality. The use of different personality guide agents to narrate the story is due to the fact that there usually exist multiple interpretations of the same historical event, depending on the storyteller's perspective [1]. The guide tells stories based on his or her past experiences taking into consideration the user's interests. The hypothesis here is that an emotional agent with personality can make interaction more realistic and natural as well as present the user with a more engaging and memorable visit by holding attention and helping comprehension of new information.

\section{Related Work}

Research in the field of Augmented Reality, Mobile and Context-Aware Tour Guide applications is growing. Cyberguide [2] is a series of prototypes of a mobile context-aware tour guide that detects the user's physical location and crude orientation, without taking into consideration the user's interests. HyperAudio [3] and HIPS [4] adopt multimodality to get round the static constraints of the environment and perform user modeling based on the history of interaction, visitor attitude, physical environment and visiting path. MARS [5] is a testbed that allows indoor and outdoor users to access and manage real world spatial information while DEEP MAP [6] project is able to generate personal guided walks through the City of Heidelberg. 
While none of the above systems employ a life-like animated character, CMAP [7], is an early attempt to build a personal mobile life-like assistant that provides visitors touring museums and open exhibitions with information based on their location and individual interests. PEACH [8] enhanced the appreciation of cultural heritage through the development of a personal guide. [9] attempts to shape dialogue interactions between an interactive gesture-choreographed conversational character and the user in an online virtual exhibition of a XVI century Portugese ship.

[10] proposed storytelling from a virtual guide perspective, constructing stories by improvising. In Geist [11], the history of the City of Heidelberg, Germany and the Thirty Years War is shown in a way that the audience receives an immersive, dramatic and action rich experience with a high factor of enjoyment. The [12] Ghost Project achieved high quality agent-based assistance without demanding visualization requirements. Some other related works are the SAGRES [13], Kyoto Tour Guide project [14], etc.

All these systems aim to provide user with context-aware information. However, the life-like animated characters employed lack a real affective model. Additionally, affective interaction between the characters and user is missing! According to [15], the individual's interaction with computers is inherently natural and social, hence affective communication is expected by people when they interact with computers. The inclusion of this missing element is the core focus of this research.

There has been a series of efforts for making artifacts with their own emotional structure. The Oz project $[16,17]$ aimed at producing agents with a broad set of capabilities where individual Woggles had specific habits and interests which were shown as different personalities. Cañamero [18] proposed an architecture that relies on both motivations and emotions to perform behavior selection that influences the creatures perception of both the external world and their own body.

Velásquez's robot, Yuppy [19] is a biologically plausible computational framework for Emotion-Based Control. Previous emotional experiences form an emotional memory, which affects action selection strategy when it re-encounters similar situations. In AlphaWolf [20], the wolves' emotions generate context-specific emotional memories based on the "somatic marker hypothesis" presented by Damasio [21], which affects how they will interact in the future.

The 'Psi' agents $[22,23]$ framework focuses on emotional modulation of perception, action-selection, planning and memory access. [24] integrates a connectionist cognitive model of emotional processing called SESAME [25] with a synthetic force model, SOF-Soar architecture [26] for training in a battlefield simulation.

\section{The Affective Guide System}

The Affective Guide System integrates the PDA with a Global Positioning System, a speech synthesis system and utilizes wireless communication. Fig. 1 gives 
an illustration of the system architecture. It consists of two emotional agents with attitude. These guides present users with different versions of the same story about an event or place allowing them to understand an event more deeply, a learning strategy targeted by the UK National Curriculum for History [27].

A brief ice-breaking session takes place before a tour starts. Based on the user's input, the guide plans a route. On the way to the destination, the guide will draw the users attention to landmarks, and applying a story-telling technique [10] will links the memory and interests of the guide as well as the visitor to the spatial location. The user interacts using the graphical user interface and receives responses by means of text, graphics and audio. A server

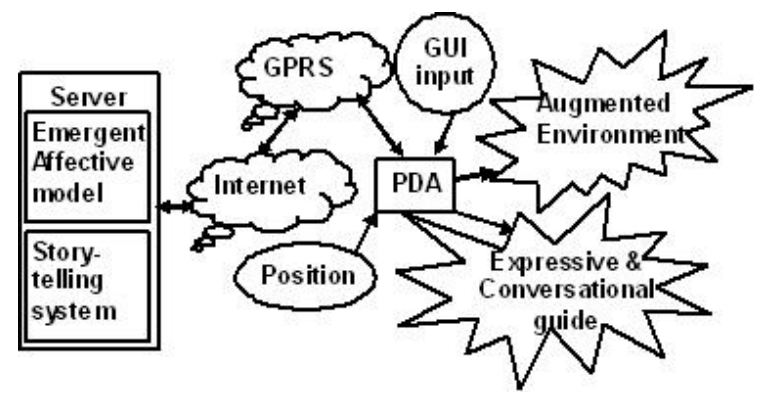

Fig. 1. The Overall System Architecture performs the processing and holds the guides emotional memory and memory about facts and sends the information to the PDA on demand.

\subsection{Emergent Affective and Personality Model}

The Emergent Affective Model, presented in Fig. 2 is designed based on the 'Psi' $[22,23]$ model with the addition of emotional memory. The 'Psi' model is unique in that emotions are not explicitly defined but emerge from the modulation of information processing, action selection, planning and memory access. Flexibility is achieved with the capability of the cognitive processes to adapt appropriately to various circumstances through the interaction of various parameters and builtin motivators.

In this model, motivation is represented by the needs and aims of the user as well as those of the guide; emotions are reflected by the modulating parameters, their causes and influences, while cognition is represented by information processes in GENERATE INTENTION, SELECT INTENTION, RUN INTENTION and PERCEPT as well as in the memory of intentions and other environmental factors.

The guide needs to maintain its level of competence (the degree of capability of coping with differing perspectives) and keeps the user attention high by adjusting its behavior appropriately to the level of uncertainty (the degree of predictability of the environment). The guide receives user inputs, system feedback and the GPS information continuously, then, generates an intention, let's say a story topic. These intentions together with its built-in motivators - level of competence and level of uncertainty are stored in a memory of intentions. The 


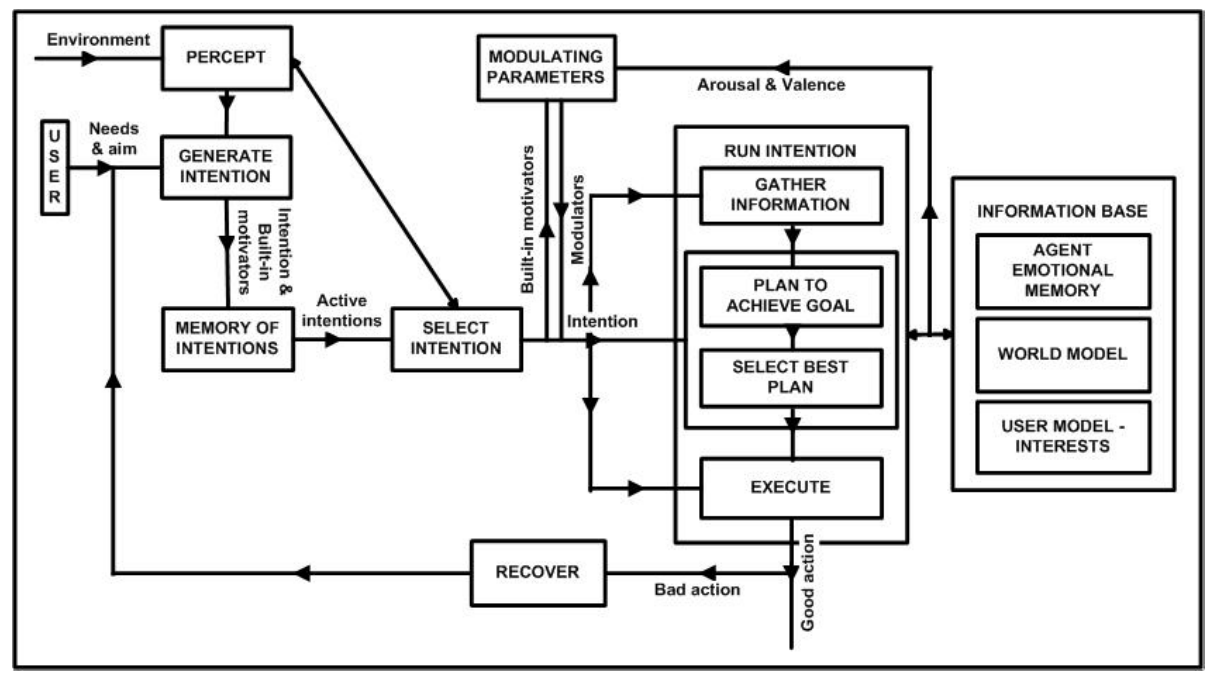

Fig. 2. The Emergent Affective and Personality Model

user's response, for example, the degree to which he or she agrees with the guide's argument, contributes to the guide's competence level, while the accuracy of the GPS reading contributes to its level of uncertainty.

Next, depending on the importance of the need and the urgency for realization, one of the active intentions is selected. Then, the guide decides autonomously whether to gather information, to design a plan or to run an existing plan. The decision is made based on the value of the built-in motivators and modulators such as arousal level (rate of processing), resolution level (carefulness and attentiveness of behavior) and selection threshold (how easy is it for another motive to take over) or in other words, the agent's current emotional state.

For example, in an uncertain environment (GPS accuracy is low) and a low level of competence (user is in disagreement with the guide's perspective), the guides arousal level and selection threshold will be high while its resolution level should be low in which case, we may diagnose that the guide is experiencing anxiety. In this situation, the agent tends to give a more general story of the current site. On the other hand, when its level of competence is high and the environment is stable, it may experience a high, but not too high level of arousal and selection threshold, with a medium resolution level. This time, the agent may be said to experience pride and hence, it is not easy for another goal to take over. It will perform some planning and provide a more elaborated story on the current subject.

By doing so, it adapts its behavior according to its internal states and the environmental circumstances. Each execution of intention will produce a feedback into the system and recovery will be performed when necessary. 


\subsection{Emotional Memory}

Recent studies in neurology provide evidence that memory files contain not only data or information but emotions as well [28]. It has also long been known that emotionally arousing events are more likely to be later recollected than similar, neutral events [29]. Those memories are part of what makes up our personality, controls our behaviors and often produces our mood.

Taking this view, the guide possesses a long-term memory that is made up of both semantic and emotional memories. Semantic memory includes locationrelated information and the user profile while emotional memory is memory for previous experiences and will be generated through simulation of past experiences. Additionally, the guide's current memory holds information about recent processing.

Emotional information can be categorised into two dimensions: arousal, that is, how exciting or calming an experience is and valence, that is, whether an experience causes a positive or a negative impact [30]. Consequently, the guide's emotional memory holds an 'arousal' tag and a 'valence' tag. The inclusion of 'arousal' tag is analogous to the Emotional Tagging concept, according to which the activation of the amygdala in emotionally arousing events marks the experience as important [31]. The 'valence' tag serves as the basis of the guide's level of competence.

When interacting with the user, the agent will be engaged in meaningful reconstruction of its own past [32], at the same time presenting facts about the place of interest. This recollective experience is related to the evocation of previously experienced emotions through the activation of the emotion tags. These values combine with the built-in motivators values to trigger the arousal level, resolution level and selection threshold, resulting in re-experiencing of emotions, though there might be a slight variation due to the input from the user.

The user's responses form positive and negative stimuli to the agent. Based on these responses, the guide will make assumptions about the user's interest. When the user adopts the guide's perspective by agreeing, the agent's level of competence increases which leads to a more detailed explanation about the subject or related subjects. Similarly, when the user disagrees, the agent's level of competences decreases and the agent tends to focus on only the important points of the subject without much elaboration.

\subsection{Personality}

Personality plays an important role in this application. Based on a brief survey of real-world tour guides experiences, we found that factors like role, interest, experience, type of tour, guide's belief, guide's personality and visitor group can affect the presentation of information. Most guides tend to incorporate beliefs and past experiences, whether his/her own or that of others while narrating a story. Different guides have different presentation styles and some guides are more chatty than others. Visitors' age, origin, race, group size, etc. also contribute to the type of story told. 


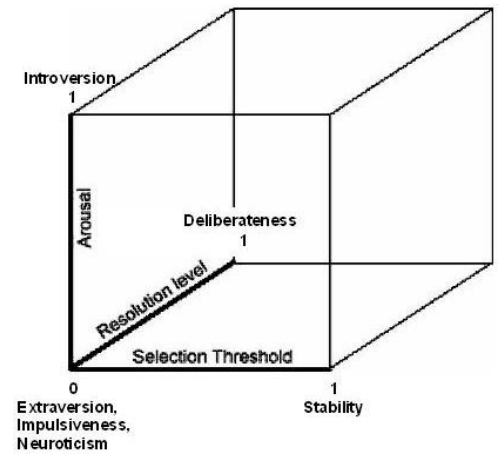

Fig. 3. Personality cube

Similarly, the virtual guide's personality will reflect the guide's ideology or perspective about a particular historical event. It manifests the guide's personal life experiences. In our model, personality emerges from varying the weight of each modulator which ranges from 0 to 1. Fig. 3 gives an illustration of how variation of the modulators' weight can lead to different personality guides.

The personality of the guide is reflected by the way it tackles interaction circumstances which map nicely to a personality traits model. The modulators are mapped onto the temperament dimensions defined by [33], where 'Psychoticism' is replaced by the Impulsivity-Deliberateness dimensions of [34] which better describe the resolution level. If a guide is given a resolution level weight of 0.1 , this will mean that the guide is impulsive. It will present the story briefly without much consideration. As the weight increases, it becomes more and more attentive and careful. When the value reaches 1 , the agent is deliberate, performs detailed planning and present stories based on the user's interest.

\section{Challenges and Future Work}

The biggest challenge of this research is retaining high user attention and generating a long-term memory. Next, is determining the relevant set of emotions. Besides that, it is important to match the appearance of the character with its behavioral and interactive potential. Appropriate representations must be established for the agent memories, the world model and the user model. The system must also take into account the special interests of each user to automatically propose an appropriate presentation of fact and story. Technically, accuracy of GPS tracking needs consideration so that the guide is spatially responsive. The limited resources of the PDA requires consideration of visual techniques other than facial animation for emotion expression.

The current focus of this research is the development of the Emergent Affective model. Since the model integrates many components, construction will be performed in a rapid prototyping manner. Evaluation is essential throughout and at the end of development phase to ensure a functional model as well as to allow refinement.

\section{Acknowledgements}

Work supported by the European Union's Sixth Framework Programme, in the IST (Information Society Technologies) Thematic Priotity IST-2002-2.3.1.6 Multimodal Interfaces, HUMAINE (Human-Machine Interaction Network on Emo- 
tion) [35] (Contract no. 507422). The authors are solely responsible for the content of this publication. It does not represent the opinion of the European Community and the European Community is not responsible for any use that might be made of data appearing therein.

\section{References}

[1] Tozzi, V.: Past reality and multiple interpretations in historical investigation. Stud Social Political Thought 2 (2000)

[2] Abowd, G.D., Atkeson, C.G., Hong, H., Long, S., Kooper, R., Pinkerton, M.: Cyberguide: A mobile context-aware tour guide. Wireless Networks 3(5) (1997) 421-433

[3] Not, E., Petrelli, D., Sarini, M., Stock, O., Strapparava, C., Zancanaro, M.: Hypernavigation in the physical space: adapting presentations to the user and to the situational context. The New Review of Hypermedia and Multimedia 4 (1998) $33-45$

[4] O'Grady, M.J., O'Rafferty, R.P., O'Hare, G.M.P.: A tourist-centric mechanism for interacting with the environment. In: Proceedings of the First International Workshop on Managing Interactions in Smart Environments, Dublin, Ireland, Springer (1999) 56-67

[5] Höllerer, T., Feiner, S., Terauchi, T., Rashid, G., Hallaway, D.: Exploring MARS: developing indoor and outdoor user interfaces to a mobile augmented reality system. Computers and Graphics 23(6) (1999) 779-785

[6] Malaka, R., Zipf, A.: Deep map challenging it research in the framework of a tourist information system. In Buhalis, D., Fesenmaier, D.R., S. Klein, e., eds.: Information and Communication technologies in tourism. Springer-Verlag, New York (2000)

[7] Sumi, Y., Etani, T., Fels, S., Simone, N., Kobayashi, K., Mase, K.: C-map: Building a context-aware mobile assistant for exhibition tours. The First Kyoto Meeting on Social Interaction and Communityware (1998)

[8] PEACH: Personal experience with active cultural heritage. (2004) http://peach.itc.it/home.htm.

[9] Almeida, P., Yokoi, S.: Interactive character as a virtual tour guide to an online museum exhibition. In: Proceeding of Museum and the Web 2003. (2003)

[10] Ibanez, J., Aylett, R., Ruiz-Rodarte, R.: Storytelling in virtual environments from a virtual guide perspective. Virtual Reality (2003)

[11] Braun, N.: Storytelling \& conversation to improve the fun factor in software applications. In Blythe, M.A., Monk, A.F., Overbeeke, K., Wright, P.C., eds.: Funology, From Usability to Enjoyment, Dordrecht, Kluwer Academic Publishers (2003)

[12] DELCA: Enter the world of ghosts: New assisting and entertaining virtual agents. Working paper, DELCA Ghost Project, IT University of Copenhagen (2004) http://www.itu.dk/research/delca/papers/delca_ghosts.pdf.

[13] Bertolleti, A.C., Moraes, M.C., da Rocha Costa, A.C.: Providing personal assistance in the sagres virtual museum. In: Proceeding of Museum and the Web 2001. (2001)

[14] Doyle, P., Isbister, K.: Touring machines: Guide agents for sharing stories about digital places (1999) 
[15] Nass, C., Steuer, J., Tauber, T.: Computers are social actors. In: CHI'94 Conference Proceedings, ACM (1994) 72-78

[16] Reilly, W.S., Bates, J.: Building emotional agents. Technical Report CMU-CS92-143, School of Computer Science, Carnegie Mellon University, Pittsburgh, PA, USA (1992)

[17] Bates, J.: The nature of characters in interactive worlds and the oz project (1992)

[18] Cañamero, D.: Modeling motivations and emotions as a basis for intelligent behavior. In Johnson, W.L., Hayes-Roth, B., eds.: Proceedings of the 1st International Conference on Autonomous Agents, New York, ACM Press (1997) 148-155

[19] Velásquez, J.: A computational framework for emotion-based control. In: Proceeding of the Grounding Emotions in Adaptive Systems Workshop, SAB '98, Zurich, Switzerland (1998)

[20] Tomlinson, B., Blumberg, B.: AlphaWolf: Social learning, emotion and development in autonomous virtual agents (2002)

[21] Damasio, A.R.: Descartes' Error: Emotion, Reason, and the Human Brain. G.P. Putnam, New York (1994)

[22] Bartl, C., Dörner, D.: Comparing the behavior of psi with human behavior in the biolab game. In Ritter, F.E., Young, R.M., eds.: Proceedings of the Second International Conference on Cognitive Modeling, Nottingham, Nottingham University Press (1998)

[23] Dörner, D.: The mathematics of emotions. In Frank Detje, D.D., Schaub, H., eds.: Proceedings of the Fifth International Conference on Cognitive Modeling, Bamberg, Germany (2003) 75-79

[24] Randolph M. Hones, Amy E. Henninger, E.C.: Interfacing emotional behavior moderators with intelligent synthetic forces. In: Proceeding of the 11th CGF-BR Conference, Orlando, FL (2002)

[25] Chown, E.: Consolidation and Learning: A Connectionist Model of Human Credit Assignment. PhD thesis, University of Michigan (1993)

[26] G. Taylor, F.K., Nielsen, P.: Special operations forces ifors. In: Proceeding of the 10th Conference on Computer Generated Forces and Behavioral Representation, Norfolk, VA (2001) 301-306

[27] NHC: History: The level description. http://www.ncaction.org.uk/subjects/history/levels.htm.

[28] Carver, J.M.: Emotional memory management: Positive control over your memory. Burn Survivors Throughout the World Inc. (2005) http://www.burnsurvivorsttw.org/articles/memory.html.

[29] Riesberg, D., Heuer, F.: Remembering the details of emotional events. Affect and Accuracy in Recall: Studies of 'Flashbulb' Memories (1992) 162-190

[30] Kensinger, E.A., Corkin, S.: Two routes to emotional memory: Distinct neural processes for valence and arousal. PNAS 101(9) (2004) 3310-3315

[31] Richter-Levin, G., Akirav, I.: Emotional tagging of memory formation - in the search for neural mechanisms. Brain Research Reviews 43 (2003) 247-256

[32] Dautenhahn, K.: The art of designing socially intelligent agents - science, fiction and the human in the loop (1998)

[33] Eysenck, H.J., Eysenck, M.: Personality and Individual Differences: A Natural Science Approach. Plenum Press, New York (1985)

[34] Buss, A.H., Plomin, R.: A temperament theory of personality development. Wiley, New York (1975)

[35] HUMAINE: Human-machine interaction network on emotion. http://emotion-research.net. 